\title{
Effects of Short-Term Plyometric Training on Physical Performance in Male Handball Players
}

\author{
by \\ Krzysztof Mazurek1, Piotr Zmijewski², , Hubert Makaruk4, Anna Mróz', \\ Anna Czajkowska ${ }^{5}$,Katarzyna Witek ${ }^{1}$, Stawomir Bodasińskit, Patrycja Lipińska
}

The aim of this study was to compare the effects of plyometric and jump training on physical performance in young male handball players. Twenty-six young male handball players were divided into two sub-groups to perform a five-week pre-season training programme supplemented with two ground-reactive protocols with an equal number of jumping exercises referred as to ground contacts: plyometric training $(P L Y ; n=14)$ and standard jump training (CON; $n=12)$. Before and after training, repeated sprint ability $(R S A)$, jumping ability (JA), maximal oxygen uptake (VO2max) and aerobic power at the anaerobic threshold (PAT) were measured. A two-factor analysis revealed significant time effects with improvements in fat mass $(p=0.012)$, maximal power during the incremental cycling test $(p=0.001)$ and PAT $(p<0.001)$, power decline $(P D E C)$ and maximal power (Pmax) in the 5 th repetition $(p<0.05$ and $p<0.01$, respectively). The training-induced changes in absolute and relative peak power in the RSA test and absolute VO2max approached significance $(p=0.06, p=0.053$ and $p=0.06$ ). No intervention time $\times$ exercise protocol effects were observed for any indices of JA, RSA and aerobic capacity. A five-week pre-season conditioning programme supplemented with only 15 sessions of plyometric exercise did not induce any additional benefits, compared to a matched format of standard jump training, in terms of improving jumping performance and maximal power in the RSA test. Aerobic capacity and the fatigue index in RSA were maintained under these two training conditions.

Key words: plyometrics, jumping, youth, repeated sprint ability, team sport.

\section{Introduction}

Team handball is classified as a highintensity, body-contact sport that demands a high level of aerobic and anaerobic fitness. For handball players, successful match performance requires several physical attributes such as speed, power, strength and agility, plus the ability to maintain performance during repeated sprints (Michalsik et al., 2013).

Training for most sports requires different physical qualities to be emphasized across an annual period, with the overall goal to improve sports performance during competition. During the pre-season period, handball training emphasizes the development of physical fitness, whereas the in-season period is aimed at maintaining aerobic and anaerobic capacity and improvement of tactical and technical skills. As a result, one of the aims of pre-season training is to improve jumping ability, repeated sprint ability (RSA) and aerobic endurance.

\footnotetext{
1 - Department of Physical Education, Józef Pilsudski University of Physical Education in Warsaw, Poland.

2 - Institute of Sport - National Research Institute, Warsaw, Poland.

3 - Research and Development Center "Legia Lab", Legia Warszawa S.A., Warsaw, Poland.

4 - Faculty of Biała Podlaska, Józef Pilsudski University of Physical Education in Warsaw, Biala Podlaska, Poland.

5 - Department of Tourism and Recreation, Józef Pilsudski University of Physical Education in Warsaw, Poland.

6 - Institute of Physical Culture, Kazimierz Wielki University in Bydgoszcz, Poland.
} 
It is common practice to include plyometric exercises in a regular training programme to increase strength and explosiveness (Chelly et al., 2014) or RSA (Chtara et al., 2017). Plyometric exercises involve rapid stretching (i.e. lengthening) of a muscle immediately before a rapid concentric contraction. This combined action is commonly called the stretch-shortening cycle (SSC) (Hermassi et al., 2014). Plyometric training, when combined with a periodized strength-training programme, has been shown to increase many performance variables: vertical jump performance, acceleration, leg strength, muscular power, increased joint awareness, and overall proprioception (Slimani at al., 2016; Stojanović et al., 2016; Ziv and Lidor, 2010).

Short-term plyometric training (i.e. 2-3 sessions a week for 6-15 weeks) can change the stiffness properties of the muscle-tendon complex and improve lower-extremity strength, power and SSC muscle function in healthy individuals (Markovic and Mikulic, 2010). Nevertheless, this study did not provide definitive conclusions regarding the effects of plyometric training on more functional abilities likely to be expressed in sport (e.g. RSA performance). One of the recommended approaches is to combine plyometrics with traditional strength training (Dæhlin et al., 2016). However, there are limited scientific data regarding the effectiveness of combining regular and plyometric training.

Efficacy testing of a short-term ( $<6$ weeks) training strategy to improve sport-specific performance in youth handball players would be of significant interest to coaches, practitioners and scientists alike. Therefore, the aim of this study was to compare the effects of plyometric and strength training on physical performance variables in young male handball players. Specifically, we examined whether a five-week pre-season plyometric programme would enhance aspects of physical performance in young athletes, relative to a standard jump training regimen.

\section{Methods}

\section{Study design}

Using a two-group design, 26 young athletes were assigned randomly to a plyometric training group (PLYO; $\mathrm{n}=14$ ) or a standard training group, which served as a control (CON; $\mathrm{n}$ $=12$ ). Both interventions supplemented a normal handball training programme during the preseason period. The 5-week pre-season period of training was assumed to be standard handball training appropriate for this sports level. Trial protocols consisted of the same numbers of jumping exercises, defined as a number of ground contacts. The only difference was the nature of the plyometric (stretch-shortening cycle included) or standard jumps (stretch-shortening cycle excluded). Performance was based on repeated sprint ability, aerobic capacity and vertical jump ability. The specific measurements included somatic data, vertical jump height during a countermovement jump (CMJ) and squat jumps (SJs), lower limb power during a drop jump (DJ) test, repeated sprint ability and aerobic capacity during two separate cycle tests. These data were collected two days before training began and within three days of completing the five-week intervention.

\section{Participants}

Twenty-four, healthy male handball players (mean \pm SD: age $=20.2 \pm 2.2$ years; body height $=183.0 \pm 5.4 \mathrm{~cm}$; body mass $=86.0 \pm 9.9 \mathrm{~kg}$, training experience $=8.4 \pm 6.3$ years) from two teams were recruited for this study. All participants were competitive handball players, and were affiliated with two professional clubs competing in the second and third division of the Polish league. Institutional ethics approval from an Ethics Committee (Institute of Sport - National Research Institute, Warsaw, Poland) was given and individual written informed consent was obtained before study commencement.

\section{Training}

The training programmes for both groups included five conditioning sessions and three sport-specific team sessions per week, all performed under supervision. Individual resistance-training programmes for the upper and the lower limbs were completed twice a week. This training was supplemented with jump training, as defined by subject allocation into the PLYO and CON sub-groups. The training loads are presented in Table 1 . The type of exercises that each group performed was matched by the number of jumps and, where possible, using similar jumps. The two groups performed the same number of jumps per session, the only 
difference being the body orientations after landing. Instructions for the PLYO group were to jump as high as possible, and keep minimum ground contact time, whereas the CON group was told to jump as high as possible, relax and improve the position after landing. Rest intervals between each jumping sets lasted $2 \mathrm{~min}$.

\section{Measurements}

The initial and final performance measurements were made at the same time of day, and under the same experimental conditions, by the same group of researchers. Verbal encouragement was provided throughout all tests to ensure maximal effort.

\section{Anthropometrics}

The anthropometric profiles of subjects were taken in the morning wearing only light clothing. Height was measured with a standard stadiometer to the nearest $0.1 \mathrm{~cm}$. Body mass (BM), absolute and relative fat mass (FM) and fatfree mass (FFM) were evaluated with an eightelectrode bioelectrical impedance device (Tanita Corp., BC-418 Tokyo, Japan), while in a standing position. The body mass index (BMI) was calculated as body mass $(\mathrm{kg}) /$ height $^{2}(\mathrm{~m})$.

Aerobic capacity

Aerobic capacity was measured using an incremental protocol on a cycle ergometer (Lode Excalibur, Groningen, The Netherlands) with a calibrated volume sensor and a breath-by-breath gas analyzer (Mes $2000 \mathrm{M}$, Cracow) to determine maximal oxygen uptake $\left(\mathrm{VO}_{2 \max }\right)$, and maximal peak respiratory minute volume (VEmax). The device was calibrated in accordance with the manufacturer's instructions. The highest values of oxygen uptake, heart rate and minute ventilation maintained for $30 \mathrm{~s}$ were considered to be $\mathrm{VO}_{2 \max }$, $H_{\text {max }}$ and VEmax, respectively. The exercise started at $50 \mathrm{~W}$, increasing in steps of $50 \mathrm{~W}$ every three minutes until volitional exhaustion. Each test was completed within $24 \mathrm{~min}$. Throughout exercise, blood collection began at $2 \mathrm{~min} 30 \mathrm{~s}$ of each threeminute stage and was completed before the start of the next step. Power output at the anaerobic threshold was determined by interpolation of the data on the 4-mmol blood lactate point.

Repeated sprint ability (RSA)

RSA was assessed using a cycle ergometer (Monark). The test consisted of $5 \times 6 \mathrm{~s}$ maximal sprints every $30 \mathrm{~s}$, with the load equal to $7.5 \%$ of the athlete's body mass. The athletes were instructed to pedal at the highest possible speed in each repetition. During the 24-s recovery between sprints, the participants rested. Straps were used to secure the feet to the pedals, and each sprint was performed in the seated position. Proprietary software (MCE 5.1. JBA Staniak, Poland) recorded peak power of each repetition; average peak power in five sprints and the fatigue index were calculated. The method used to determine total work and the decrement (\%) in power and work has been described previously (Bishop et al., 2004). The cycling protocol provides both a valid (Bishop et al., 2001) and reliable test of RSA (Bishop et al., 2001). The coefficient of variation $(\mathrm{CV})$ for RSA (total work; $\mathrm{kJ}$ ) was $3.7 \%$, based on a five-week separation period (similar to the current study) in recreationally active female students (Edge et al., 2005).

Jumping ability

Vertical jump height, based on the rise of the centre of mass, was calculated from the registered reactive force using a ground-built force plate with the MVJ v. 3.4 software package ("JBA" Staniak, Poland). Each subject performed nine maximal jumps on the top of the force plate with the aim to reach peak vertical height. The jumps included three squat jumps (SJs) with arms on the hips, three countermovement jumps with an arm swing (CMJs) and three drop jumps also with an arm swing (DJs). The subjects were given rest periods of $20 \mathrm{~s}$ between the CMJ and SJ trials, with a $60 \mathrm{~s}$ rest interval separating the DJ trials. Those jumps with the highest elevation of the body's centre of mass were selected for subsequent analysis.

\section{Statistical analyses}

Data are reported as means \pm standard deviation (SD). A two-way mixed analysis of variance (ANOVA) was used for each continuous dependent variable. The independent variables included one between-subjects factor with two levels (PLYO and CON), and one within-subject factor with two levels (pre-training and posttraining). The level of statistical significance was set at $p<0.05$. Data processing and statistical evaluations were completed using SPSS version 20.0 for WINDOWS (SPSS Inc, Chicago, IL).

\section{Results}

Somatic features

The results of two-factor ANOVA for 
somatic variables revealed only one significant main time effect for relative fat mass $(p=0.012)$; the percentage of FM was reduced significantly after the intervention, but the change was not training dependant. No significant time effect or group $\times$ time interactions were found for BM, BMI and absolute FM (Table 2).

Aerobic capacity

The two-factor ANOVA revealed a main time effect for maximal power in the incremental cycling test and aerobic power (power output at the anaerobic threshold), such that these indices increased significantly across the training period (Table 3). No time effect for $\mathrm{VO}_{2 \max }$ and $\mathrm{VE}$, and

no time $x$ training interaction was observed for any indices of aerobic capacity (Table 3).
Anaerobic capacity and jumping ability

A significant time effect was found only for $P_{D E C}$ and $P_{\max }$ in the $5^{\text {th }}$ repetition $(p<0.05$ and $p<$ 0.01 , respectively). $P_{D E C}$ and $P_{\max }$ in the $5^{\text {th }}$ repetition improved significantly after the intervention. The changes in average absolute and relative peak power in the RSA test did not reach statistical significance ( $p=0.06$ and $p=0.053$ ). Significant time main effects were also found for the SJ and CMJ ( $\quad p<0.05$ and $p<0.05$, respectively), with both decreasing with training. The DJ performance did not change significantly in any group, nor were there any differences by training group. No significant group $\times$ time interactions emerged for any index of repeated sprint ability and jumping ability (Table 4).

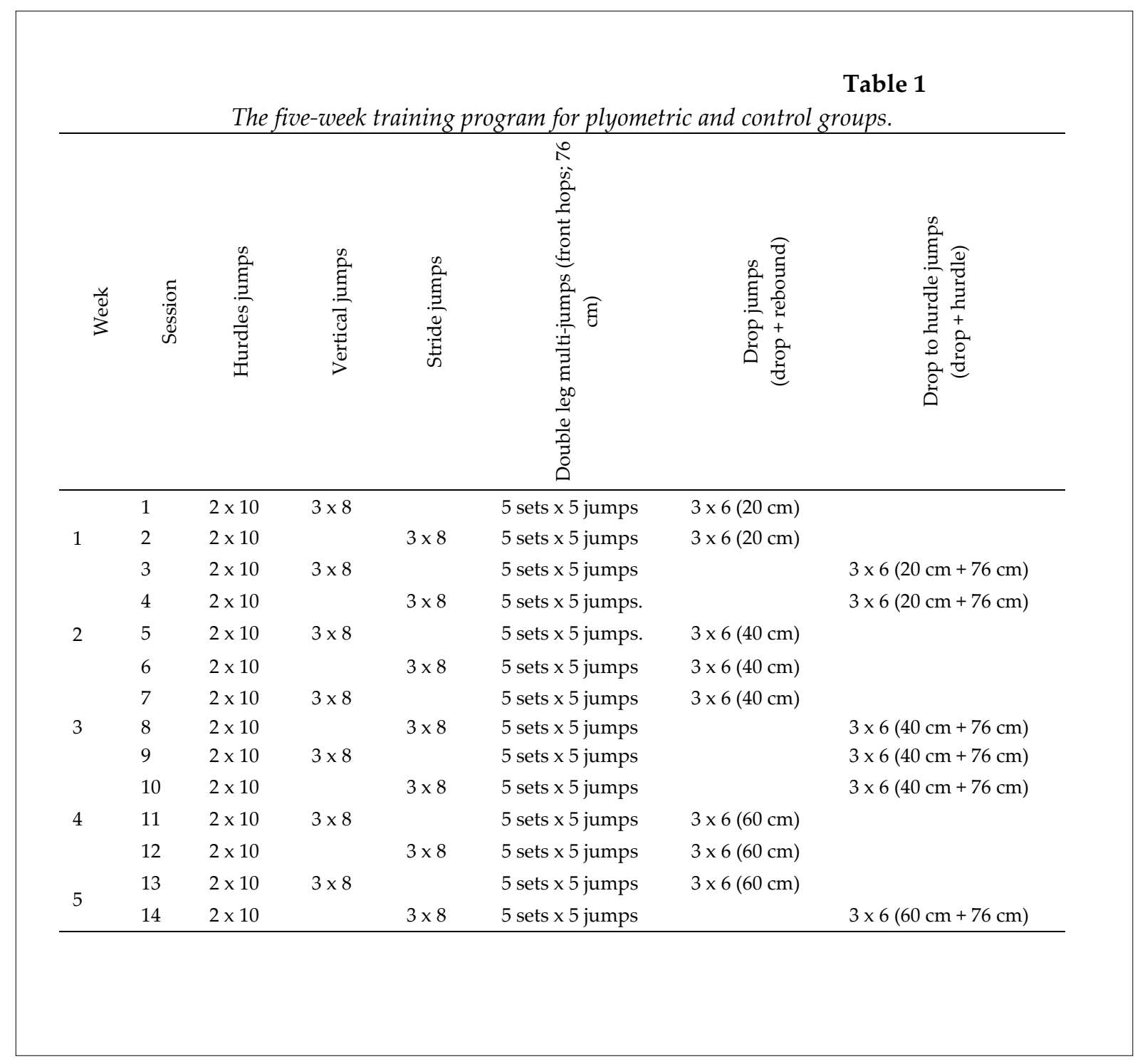


Table 2

Comparison of anthropometric indices between the plyometric training group and the control group before and after the 5-week intervention.

$\begin{array}{ccc}\begin{array}{c}\text { Control group } \\ (\mathrm{n}=12)\end{array} & \begin{array}{c}\text { Plyometric group } \\ (\mathrm{n}=14)\end{array} & \text { Effects }\end{array}$

\begin{tabular}{|c|c|c|c|c|c|c|}
\hline & Pre & Post & Pre & Post & Time & $\begin{array}{c}\text { Interaction } \\
\text { (time } x \text { exercise) }\end{array}$ \\
\hline $\begin{array}{l}\text { Body mass } \\
(\mathrm{kg})\end{array}$ & $85.6 \pm 9.6$ & $85.7 \pm 9.7$ & $86.5 \pm 10.2$ & $86.6 \pm 9.9$ & ns & ns \\
\hline $\begin{array}{l}\text { Body mass } \\
\text { index }\end{array}$ & $25.3 \pm 2.4$ & $25.2 \pm 2.3$ & $25.7 \pm 3.5$ & $25.7 \pm 3.2$ & ns & ns \\
\hline $\begin{array}{l}\text { Fat mass } \\
(\%)\end{array}$ & $15.3 \pm 2.9$ & $14.3 \pm 3.9$ & $17.0 \pm 5.0$ & $16.2 \pm 4.9$ & 0.012 & ns \\
\hline $\begin{array}{l}\text { Fat free mass } \\
(\mathrm{kg})\end{array}$ & $72.5 \pm 7.7$ & $70.9 \pm 10.6$ & $71.5 \pm 6.4$ & $72.3 \pm 6.7$ & ns & ns \\
\hline
\end{tabular}

Table 3

Comparison of aerobic capacity indices between the plyometric training group and the control group before and after the 5-week intervention.

\begin{tabular}{|c|c|c|c|c|c|c|}
\hline & \multicolumn{2}{|c|}{$\begin{array}{l}\text { Control group } \\
\quad(\mathrm{n}=12)\end{array}$} & \multicolumn{2}{|c|}{$\begin{array}{l}\text { Plyometric group } \\
\qquad(\mathrm{n}=14)\end{array}$} & \multirow{2}{*}{\multicolumn{2}{|c|}{$\begin{array}{l}\text { Effects } \\
\text { Interaction (Time } x \\
\text { Exercise) }\end{array}$}} \\
\hline & Pre & Post & Pre & Post & & \\
\hline $\begin{array}{l}\text { VEmax } \\
(1 / \min )\end{array}$ & $152 \pm 39$ & $148 \pm 34$ & $149 \pm 32$ & $154 \pm 35$ & ns & ns \\
\hline $\begin{array}{l}\mathrm{VO}_{2 \max } \\
(\mathrm{ml} / \mathrm{kg} / \mathrm{min})\end{array}$ & $45 \pm 10$ & $45 \pm 13$ & $43 \pm 5$ & $46 \pm 6$ & ns & ns \\
\hline $\begin{array}{l}\mathrm{VO}_{2 \max } \\
(1 / \min )\end{array}$ & $3.6 \pm 0.8$ & $3.8 \pm 0.9$ & $3.7 \pm 0.4$ & $4.0 \pm 0.6$ & 0.06 & ns \\
\hline $\begin{array}{l}\text { Maximal load } \\
\text { (W) }\end{array}$ & $259 \pm 33$ & $277 \pm 44$ & $261 \pm 16$ & $278 \pm 28$ & 0.001 & ns \\
\hline $\begin{array}{l}\text { Maximal load } \\
(\mathrm{W} / \mathrm{kg})\end{array}$ & $3.1 \pm 0.6$ & $3.2 \pm 0.7$ & $3.1 \pm 0.4$ & $3.3 \pm 0.5$ & 0.003 & ns \\
\hline $\begin{array}{l}\mathrm{P}_{\mathrm{AT}} \\
(\mathrm{W})\end{array}$ & $150 \pm 27$ & $168 \pm 34$ & $150 \pm 21$ & $171 \pm 36$ & 0.001 & ns \\
\hline $\begin{array}{l}\mathrm{P}_{\mathrm{AT}} \\
(\mathrm{W} / \mathrm{kg})\end{array}$ & $1.7 \pm 0.4$ & $2.0 \pm 0.5$ & $1.8 \pm 0.3$ & $2.0 \pm 0.5$ & 0.002 & ns \\
\hline
\end{tabular}


Table 4

Comparison of RSA and jumping performance indices between the plyometric training group and the control group before and after the 5-week training intervention.

\begin{tabular}{|c|c|c|c|c|c|c|}
\hline & \multicolumn{2}{|c|}{$\begin{array}{l}\text { Control group } \\
\quad(\mathrm{n}=12)\end{array}$} & \multicolumn{2}{|c|}{$\begin{array}{l}\text { Plyometric training group } \\
\qquad(\mathrm{n}=14)\end{array}$} & \multirow{2}{*}{\multicolumn{2}{|c|}{$\begin{array}{cc} & \text { Effects } \\
& \text { Interaction } \\
\text { Time } & \text { (Time } x \\
& \text { Exercise) } \\
\end{array}$}} \\
\hline & Pre & Post & Pre & Post & & \\
\hline $\mathrm{PRSA}_{\max }(\mathrm{W})$ & $853 \pm 82$ & $871 \pm 94$ & $880 \pm 107$ & $891 \pm 98$ & 0.06 & $\mathrm{~ns}$ \\
\hline $\begin{array}{l}\text { PRSA max } \\
(\mathrm{W} / \mathrm{kg})\end{array}$ & $10.1 \pm 0.9$ & $10.2 \pm 1.0$ & $10.2 \pm 0.6$ & $10.3 \pm 0.6$ & 0.053 & ns \\
\hline $\mathrm{P}_{\max 1}(\mathrm{~W})$ & $911 \pm 86$ & $917 \pm 99$ & $930 \pm 116$ & $933 \pm 105$ & ns & ns \\
\hline$P_{\max 1}(\mathrm{~W} / \mathrm{kg})$ & $10.8 \pm 1.2$ & $10.8 \pm 1.1$ & $10.8 \pm 0.8$ & $10.8 \pm 0.6$ & ns & ns \\
\hline$P_{\text {Dec }}(-)$ & $12.6 \pm 7.5$ & $10.6 \pm 4.5$ & $11.3 \pm 4.1$ & $9.3 \pm 2.3$ & 0.04 & ns \\
\hline$P_{\max 5}(\mathrm{~W})$ & $795 \pm 93$ & $812 \pm 97$ & $824 \pm 99$ & $84 \pm 96$ & 0.004 & $\mathrm{~ns}$ \\
\hline$P_{\max 5}(\mathrm{~W} / \mathrm{kg})$ & $9.4 \pm 0.9$ & $9.6 \pm 0.9$ & $9.6 \pm 0.5$ & $9.8 \pm 0.6$ & 0.004 & $\mathrm{~ns}$ \\
\hline $\mathrm{SJ}(\mathrm{m})$ & $0.40 \pm 0.08$ & $0.39 \pm 0.08$ & $0.41 \pm 0.04$ & $0.40 \pm 0.03$ & 0.039 & ns \\
\hline CMJ (m) & $0.45 \pm 0.09$ & $0.44 \pm 0.09$ & $0.47 \pm 0.06$ & $0.46 \pm 0.05$ & 0.026 & ns \\
\hline DJ (m) & $0.52 \pm 0.09$ & $0.51 \pm 0.09$ & $0.55 \pm 0.07$ & $0.56 \pm 0.06$ & ns & ns \\
\hline
\end{tabular}

\section{Discussion}

The main finding of this study was that a five-week customary pre-season training regimen (with plyometric exercises included) had a similar effect on repeated sprint ability, aerobic capacity and jumping ability as a programme that included standard jump training, when the same number of jumps were performed by young male handball players.

To date, most studies have investigated the effects of plyometric exercise added to standard training or instead of selected training loads on jumping ability, agility and endurance, yet separately. The present study is one of only a few that support data on how combining plyometric exercises and standard jumping with a regular, appropriate conditioning programme results in effectiveness of the pre-season handball training in improving simultaneously aerobic and anaerobic capacity. Beside increasing explosive power, developing aerobic fitness is an important factor influencing the ability to resist fatigue during RSA and $\mathrm{VO}_{2 \max }$ is significantly correlated 
with the RSA fatigue index in team sport athletes (Gharbi et al., 2015).

We expected a significant increase in jumping ability and sprint performance by combining regular handball training and plyometric exercises. Surprisingly, the jumping ability (SJ and CMJ) of subjects decreased in both the PLYO and CON groups. Previously, it has been shown that plyometric training can improve performance in various sports, by either combining it with regular training or using it separately to improve a specific physical component of the neuromuscular system (Slimani et al., 2016; Stojanović et al., 2016). Markovic and Mikulic (2010) reported that plyometric training, either alone or in combination with other training modalities, had the potential to enhance a wide range of performance aspects, including jumping, sprinting, agility and endurance performance, in children and young adults of both sexes. Thus, our results are contrary to several studies that have demonstrated that plyometric training can enhance muscle strength and power (Markovic, 2007a), speed (Hammami et al., 2016) and agility (Khodaei et al., 2017).

Jumping ability

We noted similar decreases in the SJ and CMJ in the PLYO and CON groups (SJ: $1.8 \%$ and 2.3\%; CMJ: $1.9 \%$ and $2.3 \%$ ). Only DJ performance increased in the PLYO group by $1.3 \%$ and there was a decrease in the CON group by $1.0 \%$, but these differences were not significant. Chelly et al. (2015) found that 10 weeks of lower limb plyometric training, added to a standard inseason regimen, increased vertical (SJ, CMJ, and DJ) as well as horizontal jump ability more than standard training. With plyometric training, absolute peak power increased by an average of $9.1 \%$, though similar gains (10\%) were achieved using a standard training approach (Chelly et al., 2015). Generally speaking, one might expect greater gains from plyometric exercises. Stojanovic et al. (2016) demonstrated positive effects on SJ, CMJ and DJ performance in females that were small, moderate to large and very large, respectively. Some studies have demonstrated no change in jump height after a six-week plyometric training programme (Gottlieb et al. 2014) and 9week training programme supplemented with plyometric exercises (Brito et al., 2014). Most authors have reported a beneficial effect (from plyometrics) with relevant improvements in jump height ranging from $4.7 \%$ (SJ and DJ), through $7.5 \%$ (CMJ with an arm swing) up to $8.7 \%$ (CMJ) (Markovic, 2007a). These results justify the application of plyometric exercises for the purpose of development of vertical jump performance in healthy individuals (Markovic, 2007a).

The conflicting reports and lack of improvements in our study participants could be explained by several factors. Slimani et al. (2016) hypothesised that plyometric training duration of 6-7 weeks was too short to improve muscular power in some elite male athletes, due to a high training level and reduced adaptive capacity. A small positive training effect of plyometrics could be observed after only 6 weeks of training (Stojanović et al., 2016). Our participants were not elite, but competed at a national and regional level and significant improvements were frequently reported in comparable populations, although the training periods exceeded 8 weeks (Slimani et al., 2016). Next, plyometric training at a lower intensity or without some progressive overloads appears to be a less effective strategy than moderately high and progressive training, respectively. The training loads in the PLYO and CON groups were both maintained, so the stimulus for adaptation was potentially reduced and/or an interference effect occurred, due to concurrent endurance training (Davitt et al., 2014; García-Pallarés and Izquierdo, 2011). Some authors have reported that, for optimising maximal strength enhancement, the combination of training modalities (i.e., plyometrics and highintensity resistance training) is needed (De Villarreal et al., 2010). In our study, jump training was executed in one mode. Previously, it was shown that a combination of plyometric drills was more effective than single plyometric drills (e.g., DJ, CMJ) (Ramírez-Campillo et al., 2015). Furthermore, a period of 10 weeks or more (more than 20 sessions of plyometric training in total) has been suggested to maximize the probability of obtaining significant performance improvements in athletes (Slimani et al., 2016). Therefore, the period of training tested in this work seems to be too short to elicit such gains.

Sprint performance

A recent meta-analysis (Slimani et al., 2016) highlighted a trend towards decreasing 
sprint times with plyometric training. In our study, we did not find significant changes in first cycle sprint performance, when measured as absolute or relative peak power, after training in either the PLYO or CON group. Similarly, Herrero et al. (2006) reported no significant gains in 20-m sprint times except when 4 weeks of plyometric training were combined with electromyostimulation. No significant differences were also observed in 5-m sprint and agility performances after a 9-week mid-season training programme supplemented with plyometric exercises (Brito et al., 2014). Many papers have reported statistically significant and positive effects of a plyometric programme on sprint performance (Arazi and Asadi, 2011; Slimani et al., 2016; Stojanović et al., 2016). In contrast, no changes were observed in selected studies. Sprint running performance did not change after 4-week plyometric training conducted on grass in 18 amateur, adult soccer players (Impellizzeri et al., 2008) as well as after 6-week depth jump (DJ) or countermovement jump (CMJ) plyometric training in semi-professional soccer players (Thomas et al., 2009).

RSA

There are only few studies investigating the effects of plyometric training on RSA in teamsport athletes. Hammami et al. (2016) revealed no significant difference in RSA variables with eightweek plyometric training incorporated into a standard soccer conditioning regimen. They found significant improvements in repeated running sprint performance in youth soccer players, but it was not more effective than traditional strength training (Hammami et al., 2016). In another study conducted on handball players, Hermassi et al. (2014) examined the effects of eight weeks of lower-limb plyometric training on shuttle running performance. They found that biweekly plyometric training improved several aspects of performance. However, no interaction effect was found between the groups performing standard training or standard training supplemented with plyometric exercises (50-100 ground contacts per session). In our study, we did not observe a significant increase in average peak power across five repetitions of all-out sprinting. The RSA scores tended to improve, but the changes did not reach statistical significance $(p=0.06)$.
Interestingly, we noted an improvement in the fatigue index and maximal power in the last sprint repetition, but there were no significant differences between the treatment groups $(p>$ 0.05).

In a previous investigation on elite youth soccer players, Buchheit el al. (2010) observed that repeated shuttle sprint training (10 weeks) improved RSA results more than explosive strength training. One reason for the lack of training-induced change herein could be poor movement specificity and lack of transfer into functional exercise. Also, training to improve sprinting power is not usually the main focus of handball practice during the pre-season phase, where emphasis is placed on the improvement of endurance and general strength.

$V O_{2 \max }$

The results of our study showed that plyometric training had no detrimental effect on endurance, or that an athlete can at least maintain a certain level of aerobic endurance while incorporating plyometric training in their regular programme. For coaches it is important whether aerobic capacity is negatively affected when handball training is performed concurrently with additional plyometric exercises. The results of our study show that the additional physiological stimulus provided by plyometric jump training was insufficient to elicit changes in cardiovascular variables in this 5 -week period. The effects of a six-week plyometric training programme on distance running performance in 17 male subjects was examined by Spurrs et al. (2003). Plyometric training improved the subjects' $3 \mathrm{~km}$ time trial performance by $1.6 \%$, but no significant changes in $\mathrm{VO}_{2 \max }$ or the lactate threshold in the experimental group were found. Therefore, it was speculated that the improved performance during the time trial was due to the improvement in running economy. We are not able to confirm this effect. The absence of $\mathrm{VO} 2$ max improvement could suggest that the duration of the training program and/or the training stimulus was insufficient to generate an improvement. This study demonstrated that although the intervention protocols did not appear to positively influence $\mathrm{VO}_{2 \max }$, they also did not hinder aerobic capacity. However, despite the lack of influence on $\mathrm{VO}_{2 \max }$, we found a significant improvement in leg power generated at the 
anaerobic threshold, similar in both groups, which could be evaluated as a positive effect of training independent of the kind of additional jumping/plyometric exercises. The other studies demonstrated that plyometric training could be successfully employed to improve running speed, running economy and selected upper and lower body strength and power measures (Arazi et al., 2012; Saunders et al., 2006). All of these fitness components are important for the majority of sports, albeit to varying degrees. Most studies, however, suggest that plyometric training may enhance overall athletic performance.

Most of the studies on the effects of plyometric training have demonstrated improvements in vertical jump performance. In contrast, a number of authors failed to report significant positive effects of plyometric training on vertical jump height, and some of them even reported negative effects (Luebbers et al., 2003; Markovic, 2007b). Thus, at present, definitive conclusions regarding the effects of PT on vertical jump performance cannot be drawn. The factors that may be responsible for the discrepancy among the results of this and other studies concern the training programme design (type of exercises used, training duration, training frequency, volume and intensity of training), subject characteristics (age, gender, fitness level) and methods of testing physical performance. Moreover, the effects of PT may differ depending on sports activity and familiarity with PT, and also the combination of unilateral and bilateral jump drills seems more advantageous to induce significant sprint performance improvements during high-intensity short-term plyometric training (Slimani et al., 2016). Plyometric exercise mainly affected the fast-twitch muscle fibres, damaging both the sarcolemma and the sarcomere at the site of the Z-disk. Thus, coaches should avoid prescribing high-volume plyometric exercise bouts within short succession or after other forms of high-intensity exercise that are known to stress the fast-twitch muscle fibres, so that athletes have sufficient time to regenerate damaged fibres (Macaluso et al., 2012). Regarding neuromuscular performance, plyometric exercises can even be performed after strength-power training on the same day if a minimum rest period of $3 \mathrm{~h}$ is provided (Hartmann et al., 2015). An adequate progressive increase in the plyometric training volume-based load should result in greater performance adaptations in explosive movements and aerobic endurance (Ramírez-Campillo et al., 2015). According to Potach and Chu (2000), plyometric programmes ranging between six and ten weeks, with training sessions two to four times per week, are sufficient to elicit positive training effects. Therefore, the number of sessions used in this study seems to be insufficient to obtain the necessary results. It could be speculated that the detraining period in the examined teams was too long and athletes did not present sufficient strength levels to perform movements like plyometrics. This situation could have delayed the adaptation period of the subjects when the plyometric intervention started, resulting in a much slower progression in exercise performance. Contrary to that, it was shown that a training programme of two weeks with three sessions per week including high-intensity plyometric exercises (between 180 and 250 jumps per session) in well-trained athletes can be recommended as a short-term strategy that will optimize the likelihood of obtaining significant improvements in explosive power and sprint velocity performance (Maćkała and Fostiak, 2015).

\section{Limitations}

There are some limitations of this study. The cycle ergometer test has been previously widely used to evaluate adaptation to physical exercise training, but does not involve the stretchshortening cycle, which is widely represented in the plyometric training programme. This could limit the possibility of detecting changes in sprint performance. In addition, sprint cycling is somewhat unfamiliar to handball players. However, the examined athletes were previously frequently tested in different anaerobic cycling tests. It should be acknowledged that our subjects were limited to one category of youth male handball players, and these observations should be extended to the female population with caution. Furthermore, more investigations are needed with different intensities and volumes of plyometric training to determine the optimum load for this form of pre-season training. It should be noted that an on-court (including plyometric actions) training programme supplemented with plyometric strength training may produce excessive physical loads for the adaptive 
capabilities of young athletes and, with the lack of a supercompensation phase, it could have a negative influence on subsequent measurements. Another limitation is the lack of an additional control group practising handball without additional jumping or plyometric drills - this could help to determine if there was any interference effect of concurrent training on endurance and explosive strength performance.

\section{Practical implications}

The study showed that plyometric training and jump training added to standard handball training did not differ significantly in enhancing submaximal aerobic and anaerobic performance of young handball players. Thus, coaches may have the possibility of alternating between these methods to maintain performance (Gottlieb et al., 2014). It could be speculated that the pre-season training period should be longer than 5 weeks to produce adequate training- induced changes in aerobic and anaerobic capacity.

\section{Conclusions}

A 5-week standard training programme supplemented with only 15 sessions of plyometric exercises seems too short to gain significant and concurrent improvements in jumping, repeated sprint ability and aerobic capacity. With the pattern of standard handball training supplemented with plyometric training that we adopted, and perhaps because participants were in good initial physical condition, only aerobic capacity and indices of fatigue in anaerobic exercise were improved, whereas indices of maximal aerobic and anaerobic capacity remained unchanged. Further studies are needed to determine how to manipulate training volume and modality during the pre-season period to maximize performance in young team sport athletes.

\section{Acknowledgements}

This study was supported by the Ministry of Science and Higher Education of the Republic of Poland by research grant 0018/RS2/2013/52 (AWF; PB-83).

\section{References}

Arazi H, Asadi A. The effect of aquatic and land plyometric training on strength, sprint, and balance in young basketball players. J Hum Sport Exerc, 2001; 6: 101-111

Arazi H, Asadi A, Nasehi M, Delpasand A. Cardiovascular and blood lactate responses to acute plyometric exercise in female volleyball and handball players. Sport Sci Health, 2012; 8: 23-29

Bishop D, Edge J, Goodman C. Muscle buffer capacity and aerobic fitness are associated with repeatedsprint ability in women. Eur J Appl Physiol, 2014; 92: 540-547

Bishop D, Spencer M, Duffield R, Lawrence S. The validity of a repeated sprint ability test. J Sci Med Sport, 2001; 4: 19-29

Brito J, Vasconcellos F, Oliveira J, Krustrup P, Rebelo A. Short-term performance effects of three different low-volume strength-training programmes in college male soccer players. J Hum Kinet, 2014; 40: 121128

Buchheit M, Mendez A, Delhomel G, Brughelli M, Ahmaidi S. Improving repeated sprint ability in young elite soccer players: repeated shuttle sprints vs. explosive strength training. J Strength Cond Res, 2010; 24: $2715-2722$

Chelly MS, Hermassi S, Aouadi R, Shephard RJ. Effects of 8-Week In-season Plyometric Training on Upper and Lower Limb Performance of Elite Adolescent Handball Players. J Strength Cond Res, 2014; 28: 1401-1410

Chelly MS, Hermassi S, Shephard RJ. Effects of In-Season Short-term Plyometric Training Program on Sprint and Jump Performance of Young Male Track Athletes. J Strength Cond Res, 2015; 29: 2128-36

Chtara M, Rouissi M, Haddad M, Chtara H, Chaalali A, Owen A, Chamari K. Specific physical trainability in elite young soccer players: efficiency over 6 weeks' in-season training. Biol Sport, 2017; 34: 137-148

Dæhlin TE, Haugen OC, Haugerud S, Hollan I, Raastad T, Rønnestad BR. Improvement of Ice Hockey 
Players' On-Ice Sprint With Combined Plyometric and Strength Training Sprint. Int J Sports Physiol Perform, 2017; 12: 893-900

Davitt PM, Pellegrino JK, Schanzer JR, Tjionas H, Arent SM. The effects of a combined resistance training and endurance exercise program in inactive college female subjects: does order matter? J Strength Cond Res, 2014; 28: 1937-1945

Edge J, Bishop D, Goodman C, Dawson B. Effects of high- and moderate-intensity training on metabolism and repeated sprints. Med Sci Sports Exerc, 2005; 37: 1975-1982

García-Pallarés J, Izquierdo M. Strategies to Optimize Concurrent Training of Strength and Aerobic Fitness for Rowing and Canoeing. Sports Med, 2011; 41: 329-343

Gharbi Z, Dardouri W, Haj-Sassi R, Chamari K, Souissi N. Aerobic and anaerobic determinants of repeated sprint ability in team sports athletes. Biol Sport, 2015; 32: 207-212

Gottlieb R, Eliakim A, Shalom A, Dello-Iacono A, Meckel Y. Improving Anaerobic Fitness in Young Basketball Players: Plyometric vs. Specific Sprint Training. J Athl Enhanc, 2014; 3: 1-6

Hammami M, Negra Y, Aouadi R, Shephard RJ, Chelly MS. Effects of an In-season Plyometric Training Program on Repeated Change of Direction and Sprint Performance in the Junior Soccer Player. J Strength Cond Res, 2016; 30: 3312-3320

Hartmann H, WirthK, Keiner M, Mickel C, Sander A, Szilvas E. Short-term Periodization Models: Effects on Strength and Speed-strength Performance. Sports Med, 2015; 45: 1373-1386

Hermassi S, Gabbett T, Ingebrigtsen J, Van den Tillaar R, Chelly M, Chamari K. Effects of a Short-Term InSeason Plyometric Training Program on Repeated-Sprint Ability, Leg Power and Jump Performance of Elite Handball Players. Int J Sports Sci Coach, 2014; 9: 1205-1216

Herrero JA, Izquierdo M, Maffiuletti NA, García-López, J. Electromyostimulation and plyometric training effects on jumping and sprint time. Int J Sports Med, 2006; 27: 533-539

Impellizzeri FM, Rampinini E, Castagna C, Martino F, Fiorini S, Wisloff U. Effect of plyometric training on sand versus grass on muscle soreness and jumping and sprinting ability in soccer players. Br J Sports Med, 2008; 42: 42-46

Khodaei K, Mohammadi A, Badri N. A comparison of assisted, resisted, and common plyometric training modes to enhance sprint and agility performance. J Sports Med Phys Fitness, 2017; 57: 1237-1244

Luebbers PE, Potteiger JA, Hulver MW, Thyfault JP, Carper MJ, Lockwood RH. Effects of plyometric training and recovery on vertical jump performance and anaerobic power. J Strength Cond Res, 2003; 17: 704-709

Macaluso F, Isaacs AW, Myburgh KH. Preferential type II muscle fiber damage from plyometric exercise. J Athl Train, 2012; 47:414-420

Maćkała K, Fostiak M. Acute Effects of Plyometric Intervention-Performance Improvement and Related Changes in Sprinting Gait Variability. J Strength Cond Res, 2015; 29: 1956-1965

Markovic G. Does plyometric training improve vertical jump height? A meta-analytical review. Br J Sport Med, 2007; 41: 349-355

Markovic G, Mikulic P. Neuro-Musculoskeletal and Performance Adaptations to Lower-Extremity Plyometric Training. Sports Med, 2010; 40: 859-895

Michalsik LB, Aagaard P, Madsen K. Locomotion characteristics and match-induced impairments in physical performance in male elite team handball players. Int J Sports Med, 2013; 34: 590-599

Potach DH, Chu DA. Plyometric training. In: Baechle TR, Earle RW, eds. Essentials of strength training and conditioning, 2nd edn., Champaign, IL: Human Kinetics, 427-470; 2000

Ramírez-Campillo R, Burgos C, Henríquez-Olguín C, Andrade DC, Martínez, C, Álvarez C, Izquierdo M. Effect of unilateral, bilateral, and combined plyometric training on explosive and endurance performance of young soccer players. J Strength Cond Res, 2015; 29: 1317-1328

Ramírez-Campillo R, Henríquez-Olguín C, Burgos C, Andrade DC, Zapata D, Martínez C, Izquierdo M. Effect of Progressive Volume-Based Overload During Plyometric Training on Explosive and Endurance Performance in Young Soccer Players. J Strength Cond Res, 2015; 29: 1884-1893

Saez-Saez de Villarreal E, Requena B, Newton RU. Does plyometric training improve strength performance? A meta-analysis. J Sci Med Sport, 2010; 13: 513-522 
Saunders PU, Telford RD, Pyne DB, Peltola EM, Cunningham RB, Gore CJ, Hawley JA. Short-Term Plyometric Training Improves Running Economy in Highly Trained Middle and Long Distance Runners. J Strength Cond Res, 2006; 20: 947-954

Slimani M, Chamari K, Miarka B, Del Vecchio FB, Chéour F.Effects of Plyometric Training on Physical Fitness in Team Sport Athletes: A Systematic Review. J Hum Kinet, 2016; 53: 231-247.

Spurrs R, Murphy A, Watsford M. Plyometric training improves distance running performance: a case study. J Sci Med Sports, 2002; 5: 41

Stojanović E, Ristić V, McMaster DT, Milanović Z. Effect of Plyometric Training on Vertical Jump Performance in Female Athletes: A Systematic Review and Meta-Analysis. Sports Med, 2017; 47: 975986

Thomas K, French D, Hayes PR. The effect of two plyometric training techniques on muscular power and agility in youth soccer players. J Strength Cond Res, 2009; 23: 332-335

Ziv G, Lidor R. Vertical jump in female and male volleyball players: a review of observational and experimental studies. Scand J Med Sci Sports, 2010; 20: 556-567

\section{Corresponding author:}

\section{Piotr Zmijewski}

Institute of Sport - National Research Institute

Trylogii 2/16; 01-982 Warsaw, Poland

E-mail: piotr.zmijewski@insp.waw.pl 